\title{
Evaluación de la calidad de vida en personas con discapacidades significativas: aplicación de la Escala San Martín en la Fundación Obra San Martín
}

\section{Evaluating the quality of life of people with profound and multiple disabilities: Use of the San Martín Scale at the Obra San Martín Foundation}

\section{Resumen}

La Escala San Martín es un instrumento que permite evaluar la calidad de vida de personas con discapacidades significativas con evidencias adecuadas de fiabilidad y validez. En 20I 2 se administró la Escala San Martín a 85 personas con discapacidad intelectual que acudían a alguno de los centros de la Fundación Obra San Martín. El presente artículo recoge los resultados obtenidos agregados en el mesosistema, un ejemplo del microsistema y las líneas de trabajo puestas en marcha a partir de los resultados obtenidos.

\section{Palabras clave}

Calidad de vida, discapacidad intelectual, discapacidades múltiples y profundas, evaluación, apoyos.

\section{Abstract}

The San Martín Scale is an instrument used to measure the quality of life of people with significant disabilities with adequate levels reliability and validity. In 20I 2, the San Martín Scale was administered to 85 adults with intellectual disabilities who were provided supports at Obra San Martin Foundation (Santander). In this article, we describe the results obtained at the mesosystem level, an example at the microsystem level, and future areas of work identified from the results.

\section{Keywords}

Quality of life, intellectual disability, profound and multiple disabilities, assessment, supports.
Irene Hierro Zorrilla

<i.hierro@fundacionobrasanmartin.org>

Fundación Obra San Martín

\section{Miguel Ángel Verdugo Alonso \\ <verdugo@usal.es> \\ Universidad de Salamanca}

\section{Laura Elisabeth Gómez}

Sánchez

<gomezlaura@uniovi.es>

Universidad de Oviedo

\section{Sonia Fernández Ezquerra}

<coordinacion@fundacionobrasanmartin.org>

Fundación Obra San Martín

Patricia Cisneros Fernández $<$ p.cisneros@fundacionobrasanmartin.org>

Fundación Obra San Martín

Para citar:

Hierro, I. et al. (201 5): "Evaluación de la calidad de vida en personas con discapacidades significativas: aplicación de la Escala San Martín en la Fundación Obra San Martín”, Revista Española de Discapacidad, 3 (I): 93-IO5.

Doi: <http://dx.doi.org/IO.5569/23405104.03.01.05>

Fecha de recepción: 30-07-20I4 Fecha de aceptación: IO-O4-20I 5 
1. Aplicación de la Escala San Martín en la Fundación Obra San Martín

“¿Cómo valoramos la calidad de vida de los nuestros?" fue la pregunta más repetida en una mañana de noviembre de 20I0, en el salón de actos de la Fundación Obra San Martín (Santander), por un grupo de 35 profesionales (entre ellos, psicólogos, terapeutas, educadores, trabajadores sociales y auxiliares técnicoeducativos) que asistían a un curso de formación sobre el modelo de calidad de vida impartido por el Dr. M. A. Verdugo.

Al conocer escalas de evaluación de calidad de vida como la Escala INTEGRAL (Verdugo et al., 2009) o la Escala GENCAT (Verdugo et al., 2008), los profesionales de la Fundación Obra San Martín consideraron que algunos ítems no encajaban con las personas a las que prestaban apoyos y que no recogían aspectos que podían ser cruciales para el bienestar de las personas con discapacidades significativas.

En los últimos años se han producido importantes avances en el campo de la discapacidad intelectual: nueva definición de discapacidad intelectual de la Asociación Americana sobre Discapacidades Intelectuales y del Desarrollo (American Association on Intellectual and Developmental Disabilities, AAIDD), operativización del concepto de calidad de vida propuesto por Schalock y Verdugo (2002/2003, 2008, 20I2), programas de intervención basados en evidencias, desarrollo de instrumentos de evaluación con evidencias de validez y fiabilidad, cambios en los roles de los profesionales, apertura hacia la comunidad, y otros. Se ha evolucionado así desde una concepción basada en la beneficencia a una perspectiva centrada en los derechos, en la participación e inclusión en la vida de la comunidad, en la autodeterminación y en la planificación de apoyos centrados en la personas. Se ha desplazado el énfasis a conceptos como calidad de vida, autodeterminación, funcionamiento, inclusión social y educativa, planes de apoyo individualizados, derechos humanos y conducta adaptativa (Verdugo et al., 2OII).

Todos estos avances tienen un fin último, la mejora de la calidad de vida de las personas con discapacidad intelectual. Para ello, en nuestro país, se han desarrollado recientemente diversos instrumentos de evaluación de la calidad de vida con adecuadas evidencias de fiabilidad y validez, entre ellos: (a) la Escala INICOFEAPS, dirigida a evaluar la calidad de vida de adultos con discapacidad intelectual o del desarrollo desde la perspectiva de la persona y desde la perspectiva de otra persona que la conoce bien (Verdugo et al.,2013a, 2013b); (b) la Escala Integral (Verdugo et al., 2009), que permite la evaluación integral de la calidad de vida de personas con discapacidad intelectual; (c) la Escala GENCAT (Verdugo et al., 2008a, 2008b, 2009, 2010), que permite la evaluación de la calidad de vida de usuarios de servicios sociales (entre ellos, personas con discapacidad intelectual, personas con discapacidad física, personas mayores, personas con problemas de salud mental, personas con drogodependencias y personas con VIH/SIDA); (d) la Escala FUMAT (Verdugo et al., 2009), dirigida a la evaluación de la calidad de vida de personas mayores; (e) los Cuestionarios de Evaluación de la Calidad de Vida en la Infancia (CVI-CVIP) (Sabeh et al., 2009), dirigidos a niños con y sin discapacidad; y (f) el Cuestionario de Evaluación de la Calidad de Vida de Alumnos Adolescentes (CCVA), también dirigido a estudiantes con o sin discapacidad durante la etapa de la adolescencia (Gómez-Vela y Verdugo, 2009).

Sin embargo, mientras se han producido notables avances en la evaluación de resultados personales relacionados con la calidad de vida de aquellas personas con discapacidades intelectuales y del desarrollo con mayores niveles de funcionamiento, en España no existen instrumentos adecuados para evaluar la calidad de vida de las personas con discapacidades significativas, de tal modo que los escasos instrumentos existentes se han centrado tradicionalmente en la evaluación de la calidad de los apoyos que reciben. Parece pues fácil confundir la calidad de los cuidados y los 
apoyos que las personas reciben con sus metas y deseos, especialmente cuando nos referimos a las personas con graves afectaciones que presentan con frecuencia limitaciones en la comunicación.

Autores como Zijlstra y Penning (2004) afirman que todavía existe una falta de instrumentos de evaluación con propiedades psicométricas adecuadas para esta población. Las personas con discapacidades significativas conforman un grupo heterogéneo que tiene en común distintos aspectos, como la dependencia de otras personas en la mayoría de actividades de su vida diaria. Su bajo nivel de funcionamiento intelectual y sus dificultades a la hora de comunicarse hacen muy difícil utilizar escalas de calidad de vida autoinformadas. Por lo tanto, las escalas de calidad de vida se realizan mayoritariamente mediante informes de otras personas que conocen bien a la persona con discapacidades significativas.

Las escalas de evaluación de calidad de vida publicadas hasta la fecha están destinadas a personas con discapacidad intelectual con menor nivel de necesidades de apoyo, por lo que incluyen ítems que reflejan indicadores de calidad de vida que en ocasiones no son relevantes en la vida de las personas con grave afectación (e.g., ítems relativos a la situación laboral). Además, encontramos que otros aspectos que pueden ser cruciales en su vida no están recogidos en estas escalas (e.g., movilidad, productos de apoyo, comunicación).

En el año 2005, el equipo formado por Petry, Maes y Vlaskamp hizo un primer intento de operativizar la calidad de vida de las personas con discapacidades significativas. Estas autoras partieron del modelo de calidad de vida propuesto por Felce y Perry (I995). Para ello entrevistaron a padres y personal de atención directa con objeto de comprobar si las cinco dimensiones eran relevantes para las personas con discapacidades severas y múltiples. Encontraron que más del $50 \%$ de las personas entrevistadas encontraban de forma espontánea relevantes las cinco dimensiones. Cuando se les preguntaba de forma directa, entre el $80 \mathrm{y} \mathrm{el}$ I०० \% de personas las encontraba relevantes.
Como resultado de las entrevistas, desarrollaron un banco de 223 ítems, estructurado en torno a las cinco dimensiones, con sus correspondientes indicadores. Para comprobar la utilidad y validez del banco de ítems, en 2007, se realizó un estudio Delphi de dos rondas con 45 expertos que evaluaron el contenido y la estructura del banco de ítems. El resultado preliminar fue un conjunto de I05 ítems divididos en seis subescalas: bienestar material, bienestar físico, bienestar social, comunicación e influencia, desarrollo personal y actividades.

En 2009, realizaron un estudio para valorar las propiedades del instrumento desarrollado a partir del estudio Delphi anterior, denominado QOL-PMD. Para ello, se evaluó la calidad de vida de 49 personas con discapacidades significativas mediante I 47 informantes (Petry et al., 2009a, 2009b). Aunque el tamaño de la muestra no era lo suficientemente grande como para ser representativo, se redujo la escala casi en un $50 \%$ (se eliminaron 50 de los IO5 ítems).

En junio de 20I I la Fundación Obra San Martín y el INICO firmaron un convenio para la elaboración de una escala de calidad de vida para personas con discapacidades significativas: la Escala San Martín, que está llamada a llenar el vacío existente en lo relativo a herramientas adaptadas y específicas para estas personas. El proceso de creación de la escala piloto constó de un estudio Delphi realizado por I 2 jueces expertos, seleccionándose aquellos que obtuvieron una media igual o superior a $3 \mathrm{y}$ una desviación típica inferior a I,5. También se realizó un grupo de discusión que permitió la reformulación, modificación y explicitación de algunos de los ítems. Así la escala piloto se compuso de I 20 ítems. Esta escala piloto fue aplicada a I 770 personas con discapacidades significativas de distintas provincias españolas.

Se realizó un análisis de los índices de homogeneidad corregida (IHc) eliminándose 5 ítems cuyos valores eran inferiores a .20. De los restantes se seleccionaron aquellos con $\mathrm{IHc}$ más altos, en total I 20 ítems ( I 2 por dimensión, excepto Inclusión Social que quedó conformada por II). 
Respecto a la consistencia interna obtenida a través del coeficiente alfa de Cronbach la Escala obtuvo un valor total de .97 . La validez se comprobó a través de un análisis factorial confirmatorio (AFC) con LISREL v. 9.I. "dando lugar a índices de ajuste que indicaban la adecuación de dicha estructura: $\chi 00=2676.694$; $\mathrm{p}=.000 ; \mathrm{RMSEA}=.054 ; \mathrm{CFI}=.984 ; \mathrm{TLI}=.98 \mathrm{I}$; SRMR $=.044 "$ (Verdugo et al., 20I4b).

El presente artículo tiene como objetivo examinar los resultados obtenidos en la administración de la Escala San Martín (Verdugo et al., 20I4b) en la Fundación Obra San Martín, y valorar las implicaciones prácticas de los mismos en las prácticas profesionales y en el funcionamiento organizacional. Los resultados detallados son los agregados en el mesosistema y un ejemplo de resultados en el microsistema.

\section{Método}

\subsection{Participantes}

La Escala San Martín se administró en 2012 a un total de 85 personas con discapacidades significativas (e.g., personas con discapacidad intelectual que requieren apoyos extensos y generalizados, y en su mayoría con otras discapacidades asociadas) que recibían apoyos en uno o más de los centros de la Fundación Obra San Martín. De ellas, algo más de la mitad, el $59 \%(n=50)$ eran mujeres, frente a 35 varones, que suponían el 4I \% de la muestra. Las edades oscilaron entre 20 y 67 años (la media y la mediana se situaron en 42 años, mientras que la moda se situó en 46 ).

En cuanto a la intensidad de los apoyos prestados el $47 \%(n=40)$ de las personas recibían apoyos de tipo extenso y el $5 \mathrm{I} \%$ $(n=45)$ recibían apoyo de tipo generalizado. De las 85 personas a las que se administró la escala, 47 contaban con la evaluación de dependencia $(55 \%)$. De éstas, el $4 \%(n=2)$ tenían reconocido un Grado II, mientras que el $96 \%$ de las personas $(n=45)$ tenían reconocido un Grado III de Gran Dependencia. En cuanto al porcentaje de discapacidad reconocido, la media se situó en 82 , la mediana en 83 y la moda en 75 . El nivel de discapacidad más bajo se situó en $5 \mathrm{I} \%$ y el más alto en $99 \%$. El 91,76\% $(n=78)$ de las personas tenían reconocido un porcentaje de discapacidad igual o mayor al $66 \%$.

Otro dato que resulta importante destacar es su grado de institucionalización. De las 85 personas, sólo 7 ( $8 \%$ ) vivían en su domicilio familiar, el restante $92 \%$ vivía en alguno de los centros residenciales de la Fundación Obra San Martín. Respecto al tiempo que llevaban las personas viviendo en la Fundación Obra San Martín, la media se situó en I 9 años de estancia, encontrándose el periodo mínimo en I año y el máximo en 45 años.

Cabe destacar que 74 personas $(87 \%)$ presentaron alguna otra condición asociada a la discapacidad intelectual y $47(52 \%)$ presentaban dos o más condiciones asociadas. Las dos condiciones más frecuentes fueron epilepsia $(37,64 \%)$ y problemas de comportamiento $(35,3 \%)$. Dentro de la categoría "otras condiciones asociadas”, se incluyeron condiciones como deterioro cognitivo, enfermedad de Parkinson e hipercolesterolemia. El $80 \%$ de las personas $(n=68)$ tenía prescrito algún tipo de tratamiento farmacológico de carácter crónico. De ellas, 35 (5 I \%) recibían dos o más tipos de medicamentos. Los tratamientos farmacológicos más administrados eran antiepilépticos $(37,64 \%)$ y neurolépticos $(35,3 \%)$.

\subsection{Instrumento}

La Escala San Martín permite evaluar la calidad de vida de personas con discapacidades significativas a través de un informante que conoce bien a la persona (al menos desde hace tres meses) y que tiene oportunidades de observarla durante periodos prolongados de tiempo y en diversos contextos. La escala cuenta con una fiabilidad y validez constatadas, lo que permite recomendar su aplicación en personas 
con discapacidades intelectuales múltiples y profundas (Verdugo et al., 2013c; Verdugo et al., 2014a).

La Escala se cumplimenta con un formato de respuesta con cuatro opciones de frecuencia: siempre, frecuentemente, a veces y nunca. La escala consta de 95 ítems que se distribuyen en torno a las ocho dimensiones de calidad de vida, de modo que todas las subescalas constan de I 2 ítems, excepto la subescala inclusión social que consta de II.

Una vez corregida, la Escala San Martín proporciona puntuaciones estándar $(M=\mathrm{I} O$; $D T=3$ ) y percentiles para cada una de las ocho dimensiones de calidad de vida, así como una puntuación general, denominada Índice de Calidad de Vida ( $M=$ I००; $D T=$ I $\left._{5}\right)$. Todas estas puntuaciones se pueden representar gráficamente en un perfil de calidad de vida.

\subsection{Procedimiento}

El proceso de administración de la escala se inició en junio de 2012 y terminó en noviembre del mismo año. La Escala fue cumplimentada por nueve profesionales de la Fundación Obra San Martín adscritos a alguno de los cinco centros participantes: Centro Residencial de Atención Básica "Edificio Jado”, Centro Residencial de Atención 24 Horas "La Albarca", Centro Ocupacional "La Semilla", Centro de Día "San Martín” y Centro de Día "Jado".

De los nueve profesionales que cumplimentaron las escalas, el $78 \%$ fueron mujeres $(n=7)$ frente a dos hombres, que suponían el $22 \%$ de la muestra. Sus edades estaban comprendidas entre 27 y 55 años; la media y la mediana se situaron en torno a los 39. En cuanto a la profesión, casi la mitad eran psicólogos $(45 \%)$, maestros $(22 \%)$, educadores $(22 \%)$ y terapeutas ocupacionales ( I I \%). En cuanto a los años de experiencia en la prestación de apoyos a personas con discapacidad intelectual, oscilaba entre I y 20 años (la media se situó en ro años y la mediana en I2). Cada profesional evaluó a una media de 9,67 personas.
El tipo de relación entre el respondiente de la escala y la persona con discapacidad significativa fue en todos los casos de carácter profesional. Con respecto al tiempo de contacto con la persona evaluada, en el $5 \mathrm{I} \%$ de los casos el evaluador conocía a la persona desde hacía más de 5 años $(n=43)$, en el $36 \%$ de los casos $(n=3 \mathrm{I})$ lo hacía desde hacía 3-5 años, y en el I3 \% ( $n=\mathrm{II})$ la relación entre respondiente y la persona con discapacidad intelectual tenía un duración de I a 2 años. En ninguno de los casos la relación tuvo una duración menor a un año.

\section{Resultados}

\subsection{Resultados en el mesosistema}

En las Figuras I y 2 se muestra muestran gráficamente las puntuaciones medias obtenidas (directas y estándar) en cada una de las dimensiones en la Fundación Obra San Martín (e.g., datos agregados de las 85 personas evaluadas). Al analizar los datos agregados encontramos que ninguna de las puntuaciones medias alcanza el punto medio teórico de la escala $(M=$ Io en el caso de las puntuaciones estándar), por lo que podemos concluir que en términos generales las puntuaciones son bajas. Las dimensiones que alcanzan el percentil más alto $(P=25)$ son autodeterminación y bienestar físico. Las dimensiones que obtienen un percentil más bajo $(P=6)$ son inclusión social y relaciones interpersonales. Estos resultados ponen en evidencia que la calidad de vida de las personas con discapacidades significativas a las que se ha administrado la Escala San Martín está sustancialmente por debajo de la media obtenida por las I.788 personas con discapacidades significativas que conformaron la muestra de validación de la escala.

\subsection{Resultados en el microsistema}

El fin último por el que la Fundación Obra San Martín apostó por el proyecto de elaboración de la Escala San Martín era obtener evidencias sobre 
Figura 1. Perfil de calidad de vida de la muestra de la Fundación Obra San Martín

\begin{tabular}{|c|c|c|c|c|c|c|c|c|c|}
\hline \multicolumn{8}{|c|}{ Perfil de calidad de vida } & ICV & Percentil \\
\hline$A U$ & BE & BF & BM & $\mathrm{DE}$ & DP & IS & RI & $>128$ & 99 \\
\hline $16-20$ & $16-20$ & $16-20$ & $16-20$ & $16-20$ & $16-20$ & $16-20$ & $16-20$ & $118-121$ & 90 \\
\hline 14 & 14 & 14 & 14 & 14 & 14 & 14 & 14 & $\begin{array}{l}114-117 \\
112-113\end{array}$ & $\begin{array}{l}85 \\
80\end{array}$ \\
\hline 13 & 13 & 13 & 13 & 13 & 13 & 13 & 13 & 110-111 & 75 \\
\hline 12 & 12 & 12 & 12 & 12 & 12 & 12 & 12 & 106-107 & 65 \\
\hline 11 & 11 & 11 & 11 & 11 & 11 & 11 & 11 & $101-103$ & 55 \\
\hline 10 & 10 & 10 & 10 & 10 & 10 & 10 & 10 & $98-99$ & 45 \\
\hline 9 & 9 & 9 & 9 & 9 & 9 & 9 & 9 & $\begin{array}{l}90-91 \\
94-95\end{array}$ & $\begin{array}{l}40 \\
35\end{array}$ \\
\hline 0 & 8 & & 8 & 8 & 8 & 8 & 8 & $\begin{array}{l}92-93 \\
90-91\end{array}$ & 30 \\
\hline 7 & & 7 & & & & 7 & 7 & 86-89 & 20 \\
\hline 6 & 6 & 6 & 6 & 6 & 6 & 6 & 6 & $79-82$ & 10 \\
\hline 5 & 5 & 5 & 5 & 5 & 5 & 5 & 5 & $<70$ & 1 \\
\hline $1-41$ & $1-4$ & $1-4$ & $1-4$ & $1-4$ & $1-4$ & $1-4$ & $1-4$ & & \\
\hline
\end{tabular}

Figura 2. Resumen de puntuaciones: muestra de la Fundación Obra San Martín

\begin{tabular}{|c|c|c|c|}
\hline $\begin{array}{l}\text { Dimensiones de calidad } \\
\text { de vida }\end{array}$ & $\begin{array}{l}\text { Puntuaciones } \\
\text { directas totales }\end{array}$ & $\begin{array}{l}\text { Puntuaciones } \\
\text { estándar }\end{array}$ & $\begin{array}{l}\text { Percentiles de las } \\
\text { dimensiones }\end{array}$ \\
\hline Autodeterminación & 27,01 & 8 & 25 \\
\hline Bienestar emocinal & 29,93 & 7 & 16 \\
\hline Bienestar físico & 35,27 & 8 & 25 \\
\hline Bienestar material & 35,27 & 7 & 16 \\
\hline Derechos & 33,85 & 7 & 16 \\
\hline Desarrollo personal & 29,06 & 7 & 16 \\
\hline Inclusión social & 21,36 & 6 & 9 \\
\hline $\begin{array}{l}\text { Relaciones } \\
\text { interpersonales }\end{array}$ & 29,41 & 6 & 9 \\
\hline \multicolumn{2}{|c|}{ Puntuación estándar total (suma) } & 56 & \\
\hline \multicolumn{2}{|c|}{$\begin{array}{r}\text { Índice de Calidad de Vida } \\
\text { (puntuación estándar compuesta)) }\end{array}$} & 82 & \\
\hline \multicolumn{3}{|c|}{ Percentil del Índice de Calidad de Vida } & 11 \\
\hline
\end{tabular}


la calidad de vida de cada una de las personas con discapacidades significativas a las que presta apoyos. Disponer de un perfil de calidad de vida de cada una de las personas usuarias permite poder planificar los apoyos prestados de forma individualizada, haciendo hincapié en aquellos aspectos que la Escala San Martín pone de manifiesto como susceptibles de mejora.

El perfil de calidad de vida nos permite contrastar los resultados de calidad de vida de la persona en comparación con el resto de personas usuarias del centro al que acude y del resto de personas usuarias de la organización. Para ejemplificar cómo se realiza la interpretación de las puntuaciones individuales describimos a continuación un caso concreto.

Adela es una mujer de 46 años que presenta discapacidad intelectual de carácter profundo y necesidades de apoyo generalizado. Tiene asociados a la discapacidad intelectual problemas de comportamiento y de salud mental. Tiene reconocido un baremo de dependencia de Grado III y un porcentaje de discapacidad del $75 \%$.

Al comparar sus resultados individuales con los resultados obtenidos por la muestra (Figura 3), encontramos que su Índice de Calidad de Vida $(I C V=79)$ alcanza un percentil menor que el logrado por la muestra $(I C V=82)$, obteniendo la misma puntuación en derechos y bienestar físico, y más alta en bienestar emocional y relaciones interpersonales. La Figura 4 muestra las puntuaciones de Adela junto con las de la Fundación Obra San Martín y las del servicio en el que recibe prestación de apoyos: el Centro Residencial de Atención 24 horas "La Albarca". Sus resultados son más bajos al contrastarlos con su vivienda, destacando la diferencia que se produce en la dimensión inclusión social.

\section{Figura 3. Comparación entre las puntuaciones obtenidas por Adela y la Fundación Obra San Martín}

\begin{tabular}{|l|c|c|c|}
\hline $\begin{array}{l}\text { Dimensiones de calidad } \\
\text { de vida }\end{array}$ & $\begin{array}{c}\text { Puntuaciones } \\
\text { directas totales }\end{array}$ & $\begin{array}{c}\text { Puntuaciones } \\
\text { estándar }\end{array}$ & $\begin{array}{c}\text { Percentiles de las } \\
\text { dimensiones }\end{array}$ \\
\hline Autodeterminación & 26 & 7 & 16 \\
\hline Bienestar emocinal & 33 & 8 & 37 \\
\hline Bienestar fisico & 35 & 8 & 37 \\
\hline Bienestar material & 31 & 5 & 37 \\
\hline Derechos & 34 & 7 & 16 \\
\hline Desarrollo personal & 26 & 6 & 9 \\
\hline Inclusión social & 18 & 5 & 5 \\
\hline $\begin{array}{l}\text { Relaciones } \\
\text { interpersonales }\end{array}$ & 30 & 7 & 16 \\
\hline \multicolumn{2}{|c|}{ Puntuación estándar total (suma) } & $\mathbf{5 3}$ & \\
\hline \multicolumn{2}{|c|}{$\begin{array}{c}\text { indice de Calidad de Vida } \\
\text { (puntuación estándar compuesta)) }\end{array}$} & $\mathbf{7 9}$ \\
\hline \multicolumn{2}{|c|}{ Percentil del Índice de Calidad de Vida } \\
\cline { 1 - 1 }
\end{tabular}

\begin{tabular}{|l|c|c|c|}
\hline $\begin{array}{l}\text { Dimensiones de calidad } \\
\text { de vida }\end{array}$ & $\begin{array}{c}\text { Puntuaciones } \\
\text { directas totales }\end{array}$ & $\begin{array}{c}\text { Puntuaciones } \\
\text { estándar }\end{array}$ & $\begin{array}{c}\text { Percentiles de las } \\
\text { dimensiones }\end{array}$ \\
\hline Autodeterminación & 27,01 & 8 & 25 \\
\hline Bienestar emocinal & 29,93 & 7 & 16 \\
\hline Bienestar físico & 35,27 & 8 & 25 \\
\hline Bienestar material & 35,27 & 7 & 16 \\
\hline Derechos & 33,85 & 7 & 16 \\
\hline Desarrollo personal & 29,06 & 7 & 16 \\
\hline Inclusión social & 21,36 & 6 & 9 \\
\hline $\begin{array}{l}\text { Relaciones } \\
\text { interpersonales }\end{array}$ & 29,41 & 6 & 9 \\
\hline \multicolumn{2}{|c|}{ Puntuación estándar total (suma) } & 56 & 11 \\
\hline \multicolumn{2}{|c|}{$\begin{array}{c}\text { Indice de Calidad de Vida } \\
\text { (puntuación estándar compuesta)) }\end{array}$} & $\mathbf{8 2}$ \\
\hline \multicolumn{2}{|c|}{ Percentil del Índice de Calidad de Vida } \\
\hline
\end{tabular}

\begin{tabular}{|c|c|c|c|c|c|c|c|c|c|}
\hline \multicolumn{8}{|c|}{ Perfil de calidad de vida } & \multirow{3}{*}{$\begin{array}{c}\text { ICV } \\
>128 \\
122-128\end{array}$} & \multirow{2}{*}{$\begin{array}{c}\text { Percentil } \\
99\end{array}$} \\
\hline AU & $\mathrm{BE}$ & BF & Bм & DE & DP & is & RI & & \\
\hline $16-20$ & $16-20$ & $16-20$ & $16-20$ & $16-20$ & $16-20$ & $16-20$ & $16-20$ & & 90 \\
\hline 14 & 14 & 14 & 14 & 14 & 14 & 14 & 14 & $112-113$ & $\begin{array}{l}85 \\
80\end{array}$ \\
\hline 13 & 13 & 13 & 13 & 13 & 13 & 13 & 13 & $\begin{array}{l}110-111 \\
108-109\end{array}$ & 75 \\
\hline 12 & 12 & 12 & 12 & 12 & 12 & 12 & 12 & $106-107$ & 65 \\
\hline 11 & 11 & 11 & 11 & 11 & 11 & 11 & 11 & $101-103$ & 55 \\
\hline 10 & 10 & 10 & 10 & 10 & 10 & 10 & 10 & 98-99 & 45 \\
\hline 9 & 9 & 9 & 9 & 9 & 9 & 9 & 9 & 96-97 & 40 \\
\hline 8 & (8) & 8 & 8 & 8 & 8 & 8 & 8 & $92-93$ & 30 \\
\hline (7) & 7 & 7 & 7 & (7) & 7 & 7 & 7 & $86-89$ & 20 \\
\hline 6 & 6 & 6 & & 6 & (6) & 6 & 6 & \begin{tabular}{|l|l|}
$00-63$ \\
$79-82$
\end{tabular} & $\frac{15}{10}$ \\
\hline 5 & 5 & 5 & 5 & 5 & 5 & $(5)$ & 5 & $\begin{array}{c}71-78 \\
<70\end{array}$ & $\begin{array}{l}5 \\
1\end{array}$ \\
\hline $1-41$ & $1-4$ & $1-4$ & $1-4$ & $1-4$ & $1-4$ & $1-4$ & $1-4$ & & \\
\hline
\end{tabular}

\begin{tabular}{|c|c|c|c|c|c|c|c|c|c|}
\hline \multicolumn{8}{|c|}{ Perfil de calidad de vida } & ICV & \multirow{2}{*}{$\begin{array}{c}\text { Percentil } \\
99\end{array}$} \\
\hline AU & $\mathrm{BE}$ & BF & BM & DE & DP & is & RI & \multirow{2}{*}{$\begin{array}{c}>128 \\
122-128\end{array}$} & \\
\hline $16-20$ & $16-20$ & $16-20$ & $16-20$ & $16-20$ & $16-20$ & $16-20$ & $16-20$ & & 90 \\
\hline 14 & 14 & 14 & 14 & 14 & 14 & 14 & 14 & 112-113 & $\begin{array}{l}85 \\
80\end{array}$ \\
\hline 13 & 13 & 13 & 13 & 13 & 13 & 13 & 13 & 110-111 & 75 \\
\hline 12 & 12 & 12 & 12 & 12 & 12 & 12 & 12 & $106-107$ & 65 \\
\hline 11 & 11 & 11 & 11 & 11 & 11 & 11 & 11 & 101-103 & 55 \\
\hline 10 & 10 & 10 & 10 & 10 & 10 & 10 & 10 & 98-99 & 45 \\
\hline 9 & 9 & 9 & 9 & 9 & 9 & 9 & 9 & $96-97$ & 40 \\
\hline 8 & 8 & 8 & 8 & 8 & 8 & 8 & 8 & $92-93$ & 30 \\
\hline 7 & 7 & 7 & (7) & 7 & 7 & 7 & 7 & $86-89$ & $\begin{array}{l}25 \\
20\end{array}$ \\
\hline & & & & & & & & 83-85 & 15 \\
\hline 6 & 6 & 6 & 6 & 6 & 6 & (6) & (6) & \begin{tabular}{|l|l|}
$79-82$ \\
\end{tabular} & 10 \\
\hline 5 & 5 & 5 & 5 & 5 & 5 & 5 & 5 & $<70$ & 1 \\
\hline $1-41$ & $1-4$ & $1-4$ & $1-4$ & $1-4$ & $1-4$ & $1-4$ & $1-4$ & & \\
\hline
\end{tabular}


El fin último de la administración de la Escala San Martín es obtener resultados individuales de la calidad de vida de las personas con discapacidades significativas. Si estudiamos detenidamente los resultados de Adela encontramos que sus puntuaciones no alcanzan la media en ninguna de las dimensiones, como consecuencia su Índice de Calidad de Vida (ICV) logra un percentil 8 .

Si nos fijamos en las dimensiones en las que obtiene una puntuación más baja (bienestar material, inclusión social y desarrollo personal) y exploramos las respuestas dadas en dichas dimensiones hallamos que varios ítems reciben la puntuación "nunca": "Tiene un espacio físico con sus pertenencias personales a su alcance" (BM_40), "Aprende cosas que le hacen ser más independiente” (DP_64), "Tiene oportunidades de conocer otros entornos diferentes al lugar donde vive" (IS_73), "Disfruta de vacaciones en entornos inclusivos" (IS_74), "Participa en actividades fuera del centro con personas ajenas a su contexto de apoyos" (IS_76), "Participa en actividades inclusivas adecuadas para sus condiciones físicas y mentales" (IS_77), "Participa en actividades inclusivas que le interesan" (IS_78), "Las actividades en las que participa tienen en cuenta las instalaciones de ocio y cultura de la zona" (IS_79) y "Se toman medidas especificas para potenciar la participación de la persona en la comunidad" (IS_82).

A la luz de estos resultados, resulta necesario diseñar apoyos dirigidos a mejorar estos indicadores; principalmente habría que aunar esfuerzos para mejorar la inclusión social de Adela, ya que puntúa "nunca" en siete de los once ítems que componen esta dimensión.

\section{Discusión}

La finalidad de la Escala San Martín es "identificar el perfil de calidad de vida de la persona, con evidencias de validez y fiabilidad, 
para la puesta en marcha de prácticas basadas en la evidencia y el diseño de planes individuales de apoyo" (Verdugo et al., 20I4b). No obstante, además de ser utilizada para este objetivo, los datos recogidos mediante la aplicación de la Escala San Martín se pueden agregar con el fin de obtener información en el mesosistema (Gómez et al., 2012) y el macrosistema (véase Gómez et al., 2013).

La administración de la Escala San Martín puede considerarse el primer paso para la implantación de prácticas basadas en la evidencia en la Fundación Obra San Martín. Schalock, Verdugo y Gómez (20I I, p. 277) definen las prácticas basadas en la evidencia como:

“prácticas o intervenciones basadas en la mejor evidencia que puede obtenerse de fuentes creíbles (i.e., profesionales de los servicios que conocen bien a los usuarios y que tienen oportunidades de observar a las personas durante periodos prolongados de tiempo en varios contextos), que utilizan métodos válidos y fiables (i.e., la Escala San Martín) y que se basan en una teoría o marco conceptual claramente articulado y con suficiente apoyo empírico (i.e., el modelo de calidad de vida de ocho dimensiones propuesto por Schalock y Verdugo, 2002/2003)”.

La Escala San Martín permite la evaluación de resultados personales relacionados con la calidad de vida. Los resultados obtenidos pueden considerarse como el primer paso en el desarrollo de prácticas basadas en la evidencia para la mejora de la calidad de vida de las personas con discapacidades significativas que reciben servicios y apoyos en la Fundación Obra San Martín. Los perfiles de calidad de vida deben ser evaluados longitudinalmente para evidenciar los resultados de las prácticas sometidas a prueba. En el caso de la Escala San Martín se estima que la frecuencia debe ser cada dos años.

La participación en la financiación, construcción, administración y difusión de la Escala San Martín supone para la Fundación
Obra San Martín un punto de inflexión como organización de servicios sociales y educativos. La Fundación es una entidad con una amplia trayectoria, más de 65 años, que se ha ido transformando en función del avance de los modelos profesionales. Así, comienza a ser una entidad innovadora, que apuesta por el desarrollo de prácticas basadas en la evidencia, por profesionalizar la labor que hace y por poner de manifiesto resultados demostrables del trabajo que realiza. Cambiar es un proceso lento y no exento de dificultades pero, sobre todo, es un proceso enriquecedor, que a largo plazo consigue que los equipos se unan por el esfuerzo y las experiencias compartidas.

Además, la Escala San Martín ha supuesto que nos abramos, que dejemos de mirar hacia nosotros y difundamos cómo somos al resto de la comunidad autónoma de Cantabria y al resto de entidades prestadoras de apoyos a personas con discapacidad intelectual de España. Puede que la imagen que se tenía desde fuera de la Fundación haya cambiado pero, sobre todo, ha cambiado la imagen que tenemos desde dentro: somos un equipo de profesionales capaces de colaborar en un proyecto de investigación, formamos parte de una entidad que ha emprendido un camino de no retorno destinado a obtener una mejora real de la calidad de vida de las personas a las que prestamos apoyos, creemos en lo que hacemos y queremos hacerlo bien.

A través de la evaluación de la calidad de vida mediante la Escala San Martín se han propuesto modificaciones en los apoyos prestados de tal forma que redunden en la mejora de la calidad de vida de la personas usuarias. La información de carácter cuantitativo resulta relevante para establecer una línea base y poder hacer comparaciones en el futuro. La información cualitativa es la que ofrece orientaciones sobre las aplicaciones prácticas de mejora de los apoyos prestados.

Por todo esto, el análisis de los resultados de las 85 aplicaciones realizadas durante el año 20 I 2 supone obtener los resultados objetivos, analizar los mismos y comenzar a elaborar y poner en práctica propuestas de mejora. Los resultados 
generales obtenidos sobre la calidad de vida de las personas con discapacidades significativas a las que se ha aplicado la Escala San Martín ponen de manifiesto que ésta es más baja de lo que esperábamos. Ninguna de las puntuaciones estándar generales alcanzan la media $(P E=\mathrm{I} O)$ y la puntuación más alta se sitúa en el percentil 25 (el resto entre los percentiles 9-I6). Si analizamos los resultados individuales, sólo 6 personas obtienen un Índice de Calidad de Vida (ICV) que se sitúe por encima del percentil 50, además 66 personas obtienen un ICV por debajo del percentil 25 .

El perfil de calidad de vida refleja lo que, a priori, el equipo de profesionales esperábamos conociendo la realidad de nuestros centros y la circunstancias de las personas a las que prestamos apoyos. Debido alto grado de institucionalización que presentan las personas (una media de I9 años en la Fundación, con un mínimo de I y un máximo de 45) la escasez de red social (y, en demasiadas ocasiones, de la participación de la familia), unidas a las graves dificultades en la comunicación que presentan muchas de las personas y teniendo claro que el uso de sistemas de comunicación alternativa es un punto a mejorar, no resulta extraño que las puntuaciones más bajas sean las obtenidas en inclusión social y relaciones interpersonales. Lo mismo ocurre con la alta puntuación lograda en bienestar físico, ya que la Fundación Obra San Martín es una entidad que viene del modelo asistencial y en la que se presta especial atención a las condiciones de salud de las personas usuarias. El hecho de que la puntuación global obtenida en autodeterminación sea de las dos más altas era algo con lo que no contábamos, pero una vez obtenidos estos resultados vemos que sí concuerdan con la línea de trabajo iniciada en 2010 en todos los centros y que ha dirigido muchos esfuerzos en la prestación de apoyos alineada con el respeto a las elecciones de las personas, con el fomento de la toma de decisiones, con tener siempre presente las características, preferencias y deseos, y con la personalización de espacios, actividades, ritmos y rutinas.

$\mathrm{Al}$ analizar las respuestas dadas a cada uno de los ítems encontramos que ítems relativos a condiciones externas a la persona son respondidos con alta frecuencia "nunca" o "a veces” en más de un $80 \%$ de las ocasiones, es decir, indicadores que no dependen de las características de la persona si no que dependen del contexto en el que se encuentra:

- $\quad$ "Las personas que le proporcionan apoyos disponen de un listado de conductas observables que expresan sus estados emocionales" (BE_I3).

- "Tiene una ficha personal donde consta lo que le gusta, le tranquiliza, lo que no soportar y cómo puede reaccionar, que todo el personal conoce y debe cumplir" (BE_I9).

- “Las personas que le proporcionan apoyos ofrecen guía y apoyo acerca de su sexualidad" (BF_36).

- $\quad$ "Las personas que le proporcionan apoyos cuentan con formación en técnicas de Apoyo Conductual Positivo" (BE_2I).

- $\quad$ "Las personas que le proporcionan apoyos disponen de formación específica sobre ética y respeto de los derechos de las personas con discapacidad" (DE_49).

- "Disfruta de vacaciones en entornos inclusivos" (IS_74).

- “Tiene una ficha de apoyos personalizados que todo el personal conoce y debe cumplir" (IS_75).

- $\quad$ "Participa en actividades inclusivas adecuadas para sus condiciones físicas y mentales" (IS_77).

- "Participa en actividades sociales fuera del lugar donde recibe servicios y apoyos" (IS_8I).

- "Se toman medidas especificas para mejorar sus habilidades de comunicación” (RI_93).

- "Tiene la oportunidad de conocer a personas ajenas al contexto de apoyo" (RI_94).

- "Se toman medidas específicas para mantener y extender sus redes sociales" (RI_95).

Es decir, hay un gran número de ítems que dependen de los apoyos prestados desde la 
Fundación Obra San Martín, y no tanto de las características de las personas, por lo que será necesario diseñar y poner en marcha distintas acciones dirigidas a realizar:

- Actividades formativas en sexualidad y discapacidades significativas, ética, derechos y Apoyo Conductual Positivo.

- Procesos relativos a sintetizar todo el conocimiento que el personal de apoyo tiene sobre las personas con discapacidad intelectual en herramientas que faciliten la transferencia de toda esta información.

- Procesos destinados a la mejora de las habilidades comunicativas.

- Procesos diseñados para mejorar las relaciones interpersonales, el acceso a nuevos contextos de participación y a la inclusión real de las personas con discapacidades significativas.

Además, al analizar las respuestas dadas a cada uno de los ítems encontramos que, en menor número, hay ítems que reciben altos porcentajes (más del $80 \%$ ) de las categorías bajas de respuesta "nunca" y "a veces". Estos ítems describen indicadores que dependen de la interacción de condiciones personales y de condiciones ambientales:

- "Participa en la elaboración de su plan individual de apoyos" (AU_O2).

- “Aprende codas que le hacen ser más independiente" (DP_64).

- "Participa en actividades inclusivas que le interesan” (IS_78).

Las líneas de trabajo en este sentido tendrían que ir encaminadas a elaborar procesos de planificación de apoyos adaptados a los niveles de comprensión, fomento de actividades destinadas a la mejora de la autonomía y proporcionar nuevas experiencias que amplíen el campo de posibles intereses de la persona alineadas con el conocimiento que el personal de atención directa tiene sobre la persona.

No obstante, como toda investigación, ésta cuenta con algunas limitaciones. Una de ellas se centra en la realización de comparaciones de resultados atendiendo a variables sociodemográficas y a servicios. El reducido tamaño de la muestra (más concretamente, el reducido número de personas que compondrían cada grupo en el análisis por variables) limita el análisis de diferencias mediante contrastes paramétricos $(N=85)$. Tales análisis constituyen una línea futura de investigación que abordaremos en un futuro inmediato.

En la misma línea, otra limitación del proyecto se encuentra en el escaso número de evaluadores que participaron en la muestra $(N=9)$, por lo que la variabilidad es reducida y la subjetividad de cada uno de ellos puede estar teniendo bastante peso en el resultados final. De hecho, en aquellos centros en los que sólo participan dos evaluadores las puntuaciones son más extremas que en aquellos centros en los que intervienen mayor número de evaluadores en los que las puntaciones alcanzan valores centrales.

La principal línea futura de investigación de este proyecto tiene como objetivo general la puesta en marcha de prácticas basadas en la evidencia para la mejora de la calidad de vida de las persona usuarias en la Fundación. De este modo, tenemos previsto administrar la Escala San Martín en 20 I 5 a las mismas personas para valorar el impacto de los programas que se están desarrollando y los cambios que se están implementando a partir de este trabajo con el fin de constatar si se producen mejoras en la calidad de vida de las personas a las que prestamos apoyos. 
Felce, D. y Perry, J. (I995). "Quality of life: Its definition and measurement”. Research in Developmental Disabilities, I6(I), 5 I-74.

Gómez, L. E. et al. (2012). "An outcomes-based assessment of quality of life in social services". Social Indicators Research, 106, 8 I-93. doi: I0.I007/SI I 205-OI I-9794-9.

Gómez, L.E. et al. (2013). "The development and use of provider profiles at the organization and systems level”. Evaluation \& Program Planning, 40, 17-26. doi: го.Іог6/j. evalprogplan.20I3.05.00I.

Gómez-Vela, M. y Verdugo, M. A. (2009). CCVA: cuestionario de evaluación de la calidad de vida de alumnos adolescentes. Madrid: CEPE.

Petry, K. et al. (2005). "Domains of quality of life of people with profound multiple disabilities: The perspective of parents and direct support staff". Journal of Applied Research in Intellectual Disabilities, I8(I), 35-46.

Petry, K. et al. (2009a). "Psychometric evaluation of a questionnaire to measure the quality of life of people with profound multiple disabilities (QOL-PMD)". Research in Developmental Disabilities, 30, I326-I336.

Petry, K. et al. (2009b). "Measuring the quality of life of people with profound multiple disabilities using the QOL-PMD: First results”. Research in Developmental Disabilities, 30 , I394-I 405.

Sabeh, E. N. et al. (2009). CVI-CVIP: Cuestionario de evaluación de la calidad de vida en la infancia: manual de aplicación. Madrid: CEPE.

Schalock, R. L., Bonham G. S. y Verdugo M. A. (2008). "Quality of life conceptual and measurement framework: from concept to application in the field of intellectual disabilities". Evaluation and program planning, 3I, I8I-I90.

Schalock, R. L. y Verdugo, M. A. (2002/2003). Quality of life for human services practitioners.
Washington, DC: American Association on Mental Retardation [Traducido al castellano por M.A. Verdugo y C. Jenaro. Calidad de vida. Manual para profesionales de la educación, salud y servicios sociales. Madrid: Alianza].

Schalock, R. L. y Verdugo, M. A. (20I2). A leadership guide to redefining intellectual and developmental disabilities organizations: eight successful change strategies. Baltimore, MD: Brookes Publishing Company.

Schalock, R. L. et al. (20I I). "Evidence-based practices in the field of intellectual and developmental disabilities: An international consensus approach". Evaluation and Program Planning, 34, 273-282. 〈doi:10.IOI6/j. evalprogplan.20I0.10.004'.

Verdugo, M. A. et al. (2008a). Escala Gencat: Informe sobre la creació d'una escala multidimensional para avaluar la qualitat de vida de les persones usuàries dels serveis socials de Catalunya. Barcelona: Generalitat de Cataluña.

Verdugo, M. A. et al. (2008b). Escala Gencat. Formulari de l'Escala Gencat de qualitat de vida-Manual d'Aplicació de l'Escala Gencat de qualitat de vida. Barcelona: Generalitat de Cataluña.

Verdugo, M. A., Arias, B., Gómez L. E. y Schalock, R. L. (20IO). "Development of an objective instrument to assess quality of life in social services: Reliability and validity in Spain”. International Journal of Clinical and Health Psychology, IO(I): I05-I23. En «http://www. aepc.es/ijchp/articulos_pdf/ijchp-343.pdf).

Verdugo, M. A. et al. (2009). Evaluación de calidad de vida en personas mayores. La Escala FUMAT. Salamanca: INICO.

Verdugo, M. A. et al. (2009). Escala Integral de Calidad de Vida. Madrid. CEPE.

Verdugo, M. A. et al. (2013a). "Evaluación de la calidad de vida en personas con discapacidades 
intelectuales o del desarrollo: la escala INICOFEAPS”. Siglo Cero, 44(3), 6-20.

Verdugo, M. A. et al. (20I4a). "Measuring quality of life in people with intellectual and multiple disabilities; Validation of the San Martin Scale”. Research in Developmental Disabilities, $35,75-86$.

Verdugo, M. A. et al. (20I4b). Escala San Martín. Evaluación de la calidad de vida de personas con discapacidades significativas. Santander: Fundación Obra San Martin/ INICO. htttp:// inico.usal.es/33r/instrumentos-evaluacion/ escala-san-martin.aspx'.

Verdugo, M. A. et al. (20I3b). Escala INICOFEAPS. Evaluación integral de la calidad de vida de personas con discapacidad intelectual o del desarrollo. Salamanca: Universidad de Salamanca, Publicaciones del INICO, Colección Herramientas 7/2013.
Verdugo, M. A. et al. (20I3c). "Evaluación de la calidad de vida en personas con discapacidades significativas: la escala San Martín”. Siglo Cero, 44(4), 6-20.

Verdugo, M. A. et al. (20I I). Bases para el desarrollo de un modelo de evaluación para personas con discapacidad intelectual adultas que viven en servicios residenciales (en línea). http://sid.usal.es/idocs/F8/FDO25938/Bases_ modelo_de_evaluacixn_CdV_y_Apoyos.pdf .

Zijlstra, R. y Penning, C. (2004). “Mensen met ernstige verstandelijke en meervoudige beperkingen". En M. Kersten y D. Flikweert (Eds.): Onderzoek overgrenzen thematische rapportages en beschouwingen naaraanleiding van het 12 de IASSID, Montpellier 2004 (pp. 49-63). Utrecht: NGBZ en LKNG/NIZW. 